\title{
Burnout en estudiantes del Plan de Estudios Combinados en Medicina, Facultad de Medicina, Universidad Nacional Autónoma de México
}

\author{
Tanya Plett-Torres, Gina Martínez-Flisser, Samuel E. Gutiérrez-Barreto, Tania Vives-Varela, Alicia Hamui-Sutton, \\ Ana Flisser
}

Introducción. El Plan de Estudios Combinados en Medicina está diseñado para que alumnos sobresalientes que cursan la carrera de medicina estudien el doctorado simultáneamente. Las exigencias para culminar el posgrado involucran situaciones de estrés que podrían llevar al desgaste. El burnout es un síndrome con tres dimensiones: agotamiento emocional, cinismo e ineficacia, que pueden disminuir las expectativas de éxito, satisfacción y rendimiento académico.

objetivo. Determinar la frecuencia de burnout y sus dimensiones en estudiantes de seis generaciones del programa, así como su correlación con características sociodemográficas, académicas y hábitos.

Sujetos y métodos. Los alumnos respondieron voluntariamente un cuestionario autoaplicado y el Maslach Burnout Inventory-Student Survey. Se calculó la prevalencia de las dimensiones del burnout y se correlacionaron con variables evaluadas en el cuestionario usando la prueba exacta de Fisher.

Resultados. Los 56 participantes no presentaron burnout y un 61\% tampoco las dimensiones del síndrome. Solas o combinadas, un $27 \%$ mostró ineficacia; un $11 \%$, cinismo, y un 7\%, agotamiento. La prevalencia de dos dimensiones fue del $5 \%$. Se encontraron diferencias significativas $(p<0,05)$ para la relación con el grupo, tocar un instrumento, cantar o actuar, sitio de residencia y la violencia en el traslado.

Conclusiones. La ausencia de burnout en los participantes posiblemente refleja satisfacción y compromiso académico. La pésima relación con compañeros y la violencia en el traslado son frecuentes en alumnos con agotamiento. Es posible que tocar un instrumento, cantar o actuar constituya una respuesta al agotamiento y al cinismo, así como otras estrategias de afrontamiento no exploradas en este estudio.

Palabras clave. Burnout. Estudiantes de medicina. Hospitales. Médicos. Profesional. Universidad.

\section{Burnout in students of the Program of Combined Studies in Medicine, Faculty of Medicine, National Autonomous University of Mexico}

Introduction. Combined Studies in Medicine is a program in which outstanding students pursue a career in Medicine and simultaneously a doctorate. The requirements to complete the graduate course involve stress situations that could lead to burnout. Burnout is a syndrome with three dimensions: emotional exhaustion, cynicism and inefficiency, which can diminish the expectations of success, satisfaction and academic performance.

Aim. To determine the frequency of burnout and its dimensions in students of six generations of the program as well as its correlation with habits, sociodemographic and academic characteristics.

Subjects and methods. Students voluntarily answered a self-applied questionnaire and the Maslach Burnout InventoryStudent Survey. The prevalence of the dimensions and burnout were calculated and were correlated with variables evaluated in the questionnaire using the Fisher's exact test.

Results. The 56 participants were negative to burnout and 61\% to the dimensions; $27 \%$ had inefficiency, $11 \%$ cynicism and $7 \%$ exhaustion, combined or alone. The prevalence of two dimensions was $5 \%$. Significant differences were found $(p<0.05)$ for the relationship with colleagues, playing instruments, singing/acting, place of residence and violence on the way to the stay.

Conclusions. The absence of burnout in the participants possibly reflects satisfaction and academic commitment. The bad relationship with colleagues and the violence on their way to the stay are frequent in students with exhaustion. It is possible that playing instruments, singing or acting was a response to exhaustion and cynicism, as well as other coping strategies not explored in this study.

Key words. Burnout. Hospitals. Medical students. Physicians. Professional. University.

Secretaría de Educación Médica (S.E. Gutiérrez-Barreto, T. VivesVarela, A. Hamui-Sutton); Plan de Estudios Combinados en Medicina (T. Plett-Torres, A. Flisser); Facultad de Medicina; Universidad Nacional Autónoma de México. Consultora independiente (G. Martínez-Flisser). México DF, México.

Correspondencia: Dra. Tanya Plett Torres. Avda. Universidad, 3000. Ciudad Universitaria. Facultad de Medicina. Edif. B, 1er. piso. Secretaría General. Coyoacán. CP 04510. México DF, México.

E-mail:

plett@unam.mx

Agradecimientos:

Silvia Ortiz León, jefa del

Departamento de Psiquiatría y Salud Mental de la Facultad de Medicina de la UNAM, y la profesora Aurora Jaimes Medrano, que coordinaron la atención a los alumnos.

Recibido:

17.08.18.

Aceptado: 05.09.18.

Conflicto de intereses: No declarado.

Competing interests: None declared.

(c) 2018 FEM 


\section{Introducción}

El Plan de Estudios Combinados en Medicina (PECEM) tiene como objetivo que alumnos que cursan la carrera de médico cirujano en la Universidad $\mathrm{Na-}$ cional Autónoma de México (UNAM), con una nota media igual o superior a 9, sin asignaturas suspendidas y con vocación para la investigación, obtengan simultáneamente su título de licenciatura y el grado de Doctor en Medicina en un período de ocho años. En siete semestres cursan asignaturas matutinas de la licenciatura, diariamente una estancia de investigación y semanalmente una actividad académica de posgrado, ambas vespertinas. Posteriormente, cursan un semestre de internado clínico, otro en investigación, un año de servicio social (inicio del proyecto doctoral) y dos años y medio para la conclusión, publicación de la investigación y obtención del grado [1]. En este proceso debe mantenerse la excelencia académica.

El burnout laboral es una respuesta negativa a estresores crónicos por relaciones profesionales. El síndrome es tridimensional: agotamiento emocional, cinismo (actitud distante hacia el trabajo y las personas) e ineficacia (sensación de incompetencia profesional). Las etapas de burnout implican estrés agudo, sobreesfuerzo de adaptación, menor compromiso y absentismo, lo que genera ansiedad, baja autoestima, aislamiento, autoculpabilidad, pérdida de ideales e intentos de suicido [2-4]. La medición tridimensional del síndrome se realiza con instrumentos como el Maslach Burnout Inventory (MBI) y sus adaptaciones según la población diana, como el creado para estudiantes (MBI-SS), que evalúa la sensación de no poder dar más física y psíquicamente en los estudios (agotamiento emocional), desvalorización y pérdida de interés por el estudio (cinismo), y dudas sobre la capacidad para realizar el trabajo académico (ineficacia). En consecuencia, hay un bajo rendimiento, es difícil alcanzar objetivos y se produce deserción [3]. La prevalencia del burnout en estudiantes es variable (15-50\%), siendo más frecuente el agotamiento (20$40 \%$ ), seguido del cinismo (4-30\%) -relacionado inversamente con facilitadores académicos- y la ineficacia (20-50\%) -que también se relaciona con sexo, semestre y carrera- [2,5-14]. El objetivo del presente estudio fue determinar la frecuencia de burnout y sus dimensiones en estudiantes de las seis generaciones del PECEM, así como su correlación con características sociodemográficas, académicas y hábitos.

\section{Sujetos y métodos}

Alumnos de las seis generaciones del PECEM exis- tentes fueron invitados a participar en el estudio mediante consentimiento informado. El burnout se determinó con la versión validada en castellano del MBI-SS, interpretando los resultados de acuerdo con el Instituto Nacional de Higiene y Seguridad en el Trabajo de España (NTP-032) [14-16]. Los 15 ítems del cuestionario constaron de escala tipo Likert con una puntuación de 0 (nunca/ninguna vez), 1 (casi nunca/pocas veces al año), 2 (algunas veces/ una vez), 3 (regularmente/pocas veces al mes), 4 (bastantes veces/una vez por semana), 5 (casi siempre/algunas veces por semana) y 6 (siempre/todos los días):

- Cinco items evaluaron el agotamiento: 'las actividades académicas de esta carrera me tienen emocionalmente agotado,' 'me encuentro agotado físicamente al final de un día en la universidad', 'estoy cansado por la mañana cuando me levanto y tengo que afrontar otro día en la universidad', 'estudiar o ir a clases todo el día me causa tensión' y 'estoy exhausto de tanto estudiar'.

- Cuatro items evaluaron el cinismo: 'me he distanciado de mis estudios porque pienso que no serán realmente útiles', 'dudo de la importancia y valor de mis estudios', 'he perdido interés en la carrera desde que empecé en la universidad' y 'he perdido entusiasmo por mi carrera'.

- Seis items evaluaron la eficacia: 'puedo resolver de manera eficaz los problemas relacionados con mis estudios,' 'creo que contribuyo adecuadamente a las clases en la universidad', 'en mi opinión, soy un buen estudiante,' 'me estimula conseguir objetivos en mis estudios', 'he aprendido muchas cosas interesantes durante mi carrera' y 'durante las clases tengo la seguridad de que soy eficaz en la finalización de las cosas'.

Las puntuaciones de cada dimensión se obtuvieron sumando los puntos obtenidos por cada alumno; el resultado se dividió entre el número de ítems de la dimensión y los valores de las medias se distribuyeron en cuartiles y se categorizaron: muy bajo $(<5 \%)$, bajo (5-25\%), medio-bajo ( $>25-50 \%)$, medio-alto (>50\%-<75\%), alto ( $\geq 75 \%-95 \%)$ y muy alto (>95\%). Se consideró que un estudiante presentaba una dimensión si mostraba valores altos-muy altos ( $\geq 75 \%$ ) en agotamiento y cinismo, valores bajos-muy bajos $(\leq 25 \%)$ en eficacia (ineficacia) y burnout si mostraba las tres [17]. Se notificó el resultado al estudiante en una carta personalizada de invitación a acudir al Departamento de Psiquiatría y Salud Mental de la Facultad de Medicina de la UNAM para una intervención si lo consideraba necesario.

Se evaluaron características sociodemográficas (edad, sexo, con quién vive, financiación de los es- 
tudios, sitio de residencia, tiempo y violencia en los traslados), académicas (opción de carrera, semestre que cursa, relación con la mayoría del grupo) y de hábitos (horas de sueño al día, días y minutos de ejercicio a la semana, actividades extraescolares) mediante un cuestionario autoaplicado. Los tres documentos fueron aplicados en la misma sesión (por generación) entre febrero y julio de 2017, en etapas de mayor carga académica: la primera generación, al presentar el examen de candidatura; la segunda, al inicio del segundo semestre del servicio social; la tercera, una vez finalizando el semestre de internado clínico; la cuarta, en el noveno semestre, y la quinta y la sexta, en exámenes departamentales. Se obtuvieron medidas de tendencia central y de dispersión para las variables continuas. Edad, horas de sueño y días y minutos de ejercicio se agruparon en intervalos y se sumó el número de actividades extraescolares. Se analizaron diferencias entre los grupos por la prueba exacta de Fisher (bilateral). Diferencias significativas tuvieron un valor $p<0,05$. El análisis se realizó utilizando el programa STATA v. 11. El proyecto fue aprobado por las comisiones de investigación y de ética de la facultad (FM/D/151/2016).

\section{Resultados}

\section{Distribución de las características de la población estudiada}

La cuarta generación mostró mayor participación en el estudio (23\%) y era la más numerosa del PECEM; un $78 \%$ de los participantes tenían $\geq 21$ años y un $71 \%$ eran hombres (Tabla I). Para el $87 \%$ de los estudiantes, la carrera de medicina fue su primera opción, mientras que la licenciatura en ciencias genómicas y en investigación biomédica fueron más frecuentes como segunda o tercera opción (67\%). El $70 \%$ de los alumnos vivían acompañados, un 63\% con familiares, y ninguno vivía con su pareja. La financiación de sus estudios fue principalmente por padres y becas $(86 \%)$ y un $5 \%$ de los estudiantes trabajaban. El $82 \%$ de los alumnos refirió buena a excelente relación con su grupo y un $18 \%$ refirió que era de regular a pésima. El $70 \%$ de los participantes dormía $\leq 6 \mathrm{~h} /$ día, un $57 \%$ realizaba ejercicio 0-2 h/ día, y un 64\%, < 150 min de ejercicio a la semana. En total, se comunicaron 158 actividades extraescolares, siendo tres actividades combinadas las más frecuentes $(28 \%)$ : ir al cine $(n=37)$, socialización en reuniones o fiestas $(n=28)$ y hacer deporte o leer $(n=27)$. El $59 \%$ de los alumnos estaba soltero, el
87\% vivía en la Ciudad de México y el 13\% en el Estado de México. La mitad de los alumnos vivía en la delegación Tlalpan (29\%) o Coyoacán (21\%). Al 21\% de los alumnos les tomaba $\geq 121$ min el traslado a su estancia de investigación y el $23 \%$ comunicó violencia en el traslado.

\section{Dimensiones del burnout}

La dimensión más frecuente fue ineficacia (27\%), seguida de cinismo (11\%) y agotamiento (7\%) (Tabla II). Ningún alumno presentó burnout y un $61 \%$ no mostró dimensiones del síndrome. El 34\% mostró una dimensión, y el 5\%, dos. Las combinaciones de las dimensiones fueron agotamiento y cinismo, agotamiento e ineficacia, y cinismo e ineficacia ( $2 \%$ cada una).

\section{Dimensiones del burnout y características de la población}

La distribución de dimensiones del burnout y las características de la población se muestran en la tabla III. La ausencia de dimensiones (79\%) y la presencia de una dimensión fueron más frecuentes en alumnos $\geq 21$ años; todos los alumnos con sólo agotamiento fueron de este estrato de edad. Por el contrario, resultó más frecuente la presencia de dos dimensiones en alumnos $<20$ años, sin diferencias significativas. Los hombres representaron a la mayoría de los participantes del estudio y en ellos se encontró una frecuencia mayor no significativa de todas las dimensiones, excepto para cinismo e ineficacia, presentadas por una alumna. La cuarta (26\%) y la segunda generación (20\%) presentaron menor frecuencia de dimensiones, pero sin significación estadística. La mayor frecuencia con sólo ineficacia fue en la quinta (31\%) y segunda (23\%) generación. La misma frecuencia de sólo cinismo (25\%) se presentó en la primera, segunda, quinta y sexta generación, mientras que sólo agotamiento (50\%) fue en la tercera y cuarta generación. De los alumnos con dos dimensiones, un 67\% pertenecían a la sexta generación, y el resto, a la tercera. Para casi todos los alumnos sin dimensiones (94\%) y todos con sólo agotamiento o con dos dimensiones, la carrera de medicina fue su primera opción, al contrario que para el $25 \%$ de los alumnos con sólo cinismo y el $31 \%$ con sólo ineficacia, en los cuales no fue su primera opción (sin significación). Todos los alumnos con sólo agotamiento y el $31 \%$ con sólo ineficacia vivían solos. La mayoría de alumnos con dimensiones vivían con familiares y un alumno con sólo cinismo vivía con amigos (25\%), sin ser diferencias 
Tabla I. Distribución de las características de la población estudiada $(n=56)$.

\begin{tabular}{|c|c|c|c|c|c|c|c|}
\hline & & $n$ & $\%$ & & & $n$ & $\%$ \\
\hline \multirow{6}{*}{ Generación } & Primera & 4 & 7 & \multirow{2}{*}{$\begin{array}{l}\text { Días de ejercicio } \\
\text { a la semana }\end{array}$} & 0-2 días & 32 & 57 \\
\hline & Segunda & 11 & 20 & & $\geq 3$ días & 24 & 43 \\
\hline & Tercera & 8 & 14 & & & & \\
\hline & Cuarta & 13 & 23 & \multirow{2}{*}{$\begin{array}{l}\text { Tiempo de ejercicio } \\
\text { a la semana }\end{array}$} & $\geq 150 \min$ & 20 & 36 \\
\hline & Quinta & 10 & 18 & & $<150 \min$ & 36 & 64 \\
\hline & Sexta & 10 & 18 & \multirow{12}{*}{$\begin{array}{l}\text { Actividades } \\
\text { extraescolares del } \\
\text { alumno }(n=158)\end{array}$} & Hacer deporte & 27 & 17 \\
\hline \multirow[b]{2}{*}{ Edad } & $\leq 20$ años & 12 & & & Bailar & 8 & 5 \\
\hline & $\geq 21$ años & $\begin{array}{l}12 \\
43\end{array}$ & $\begin{array}{l}22 \\
78\end{array}$ & & $\begin{array}{l}\text { Tocar algún instrumento, } \\
\text { cantar o actuar }\end{array}$ & 13 & 8 \\
\hline \multirow{3}{*}{ Sexo } & \multirow{3}{*}{$\begin{array}{l}\text { Hombre } \\
\text { Mujer }\end{array}$} & \multirow{3}{*}{$\begin{array}{l}40 \\
16\end{array}$} & \multirow{3}{*}{$\begin{array}{l}71 \\
29\end{array}$} & & Pintar & 1 & 1 \\
\hline & & & & & Leer & 27 & 17 \\
\hline & & & & & Ir al cine & 37 & 24 \\
\hline \multirow{8}{*}{ Semestre que cursa } & & & & & Ir al teatro & 7 & 4 \\
\hline & Cuarto & 10 & 18 & & \multirow{2}{*}{$\begin{array}{l}\text { Socializar en } \\
\text { reuniones o fiestas }\end{array}$} & \multirow{2}{*}{28} & \multirow{2}{*}{18} \\
\hline & Séptimo & 10 & 18 & & & & \\
\hline & Noveno & 13 & 23 & & Ir a conciertos & 7 & 4 \\
\hline & Internado semestre 1 & 9 & 14 & & Ninguna & 3 & 2 \\
\hline & Servicio social semestre 1 & 11 & 20 & & & & \\
\hline & Investigación semestre 1 & 4 & 7 & \multirow{7}{*}{$\begin{array}{l}\text { N.o de actividades } \\
\text { extraescolares }\end{array}$} & Ninguna o una & 9 & 16 \\
\hline & & & & & Dos & 15 & 27 \\
\hline \multirow{4}{*}{$\begin{array}{l}\text { Carrera de } \\
\text { medicina como } \\
\text { opción de estudio }\end{array}$} & Primera & 49 & 87 & & Tres & 16 & 28 \\
\hline & Segunda ${ }^{a}$ & 6 & 11 & & Cuatro & 11 & 20 \\
\hline & Tercera a & 1 & 2 & & Cinco & 4 & 7 \\
\hline & & & & & Seis & 0 & 0 \\
\hline \multirow{2}{*}{ El alumno vive solo } & No & 39 & 70 & & Siete & 1 & 2 \\
\hline & Sí & 17 & 30 & \multirow[b]{2}{*}{ Estado civil } & Soltero(a) & 33 & 59 \\
\hline \multirow{6}{*}{ El alumno vive con } & Vive solo & 17 & 30 & & Tiene novio(a) & 23 & 41 \\
\hline & Familiares & 35 & 63 & \multirow{15}{*}{$\begin{array}{l}\text { Delegación } \\
\text { o estado } \\
\text { en el que vive }\end{array}$} & & & \\
\hline & Amigos & 3 & 5 & & Coyoacán & 12 & 21 \\
\hline & Pareja & 0 & 0 & & Tlalpan & 16 & 29 \\
\hline & Estudiantes & 1 & 2 & & Miguel Hidalgo & 2 & 3 \\
\hline & & 1 & 2 & & Álvaro Obregón & 2 & 3 \\
\hline \multirow{6}{*}{$\begin{array}{l}\text { Financiación de los } \\
\text { estudios del alumno }\end{array}$} & & & & & Iztapalapa & 2 & 3 \\
\hline & Beca(s) & 5 & 9 & & Xochimilco & 3 & 5 \\
\hline & Trabajo & 0 & 0 & & Venustiano Carranza & 1 & 2 \\
\hline & Padres y beca(s) & 48 & 86 & & Benito Juárez & 7 & 13 \\
\hline & Padres, beca(s) y trabajo & 2 & 3 & & Gustavo A. Madero & 1 & 2 \\
\hline & Beca y trabajo & 1 & 2 & & Azcapotzalco & 1 & 2 \\
\hline \multirow{8}{*}{$\begin{array}{l}\text { Relación que tiene } \\
\text { el alumno con la } \\
\text { mayoría del grupo }\end{array}$} & & & & & Tláhuac & 1 & 2 \\
\hline & Pésima & 2 & 3 & & Cuauhtémoc & 1 & 2 \\
\hline & Muy mala & 1 & 2 & & Estado de México & 7 & 13 \\
\hline & Mala & 1 & 2 & & & & \\
\hline & Regular & 6 & 11 & & $\leq 30 \mathrm{~min}$ & 12 & 21 \\
\hline & Buena & 34 & 61 & (ida y vuelta) al & $31-60 \mathrm{~min}$ & 14 & 25 \\
\hline & Muy buena & 0 & 0 & & $61-120 \mathrm{~min}$ & 18 & 33 \\
\hline & Excelente & 12 & 21 & investigación & $\geq 121 \mathrm{~min}$ & 12 & 21 \\
\hline Tiempo que el & $\leq 6 \mathrm{~h}$ & 39 & 70 & Violencia sufrida & No & 43 & 77 \\
\hline alumno duerme al día & $>6 \mathrm{~h}$ & 17 & 30 & durante el traslado & Sí & 13 & 23 \\
\hline
\end{tabular}

a Otra opción de carrera: ciencias genómicas e investigación biomédica, ciencias de la comunicación, matemáticas, cinematografía, biología. 
significativas. La financiación por padres y becas fue lo más frecuente en la mayoría de los participantes, pero el $50 \%$ de quienes presentaron sólo agotamiento reportaron este tipo de financiación, y la otra mitad, beca y trabajo. De los alumnos que trabajaban, el 67\% mostró agotamiento o ineficacia. Contrariamente a la mayoría de los participantes, los alumnos con cinismo y otra dimensión afirmaron dormir $>6 \mathrm{~h} /$ día, sin ser una diferencia significativa. La frecuencia de muy mala a mala relación con el grupo en alumnos sin dimensiones fue del $6 \%$. Por el contrario, la mitad de los participantes con sólo agotamiento o con agotamiento e ineficacia comunicaron una pésima relación con el grupo. Las dimensiones más frecuentes en donde la relación con el grupo resultó regular fueron sólo cinismo (25\%), sólo ineficacia (23\%) o combinadas (100\%), mostrando diferencias significativas. La mayoría de los participantes comunicaron 0-2 días de ejercicio a la semana. En contraste, la mitad de los alumnos con sólo agotamiento, la mayoría con sólo cinismo (75\%) y el alumno con cinismo e ineficacia reportaron $\geq 3$ días de ejercicio a la semana. Aunque la mayoría de los participantes dijo realizar $<150$ min de ejercicio a la semana, los alumnos con cinismo y otra dimensión comunicaron realizar $\geq 150$ min de ejercicio a la semana, sin significación estadística. Los alumnos sin dimensiones sumaron 100 actividades extraescolares; con ineficacia sumaron 30 actividades y con sólo cinismo sumaron 13 actividades. La diferencia significativa ( $p=0,004)$ encontrada fue en tocar un instrumento, cantar o actuar, más frecuente en alumnos con sólo cinismo (23\%), sólo agotamiento o también con cinismo (20\%). El cine fue la actividad más frecuente en alumnos sin dimensiones (25\%) y en aquellos con sólo ineficacia (20\%) o también agotamiento (100\%). Los estudiantes con sólo agotamiento comunicaron por igual (20\%) hacer deporte, tocar algún instrumento e ir al cine o al teatro. La actividad más frecuente entre aquellos con sólo cinismo fue leer (23\%), y con otra dimensión, leer, ir al cine o socializar. El número máximo de combinaciones de actividades extraescolares fue diferente para cada dimensión, sin tendencias significativas. Los alumnos sin dimensiones o sólo agotamiento realizaban más frecuentemente dos o tres actividades extraescolares (26\% y $100 \%$, respectivamente); los alumnos con cinismo, tres (75\%), y con ineficacia, dos (39\%); aquel con agotamiento y cinismo, cinco; con agotamiento e ineficacia, 0-1, y con cinismo e ineficacia, cuatro actividades. La mayoría de los participantes eran solteros y todos los alumnos con sólo agotamiento y combinado con ineficacia afirmaron tener novio(a), sin llegar a la significación. La ma-
Tabla II. Distribución de las dimensiones del burnout $(n=56)$.

\begin{tabular}{|c|c|c|c|}
\hline & & $n$ & $\%$ \\
\hline \multirow{2}{*}{ Ineficacia } & No & 41 & 73 \\
\hline & Sí & 15 & 27 \\
\hline \multirow{2}{*}{ Cinismo } & No & 50 & 89 \\
\hline & Sí & 6 & 11 \\
\hline \multirow{2}{*}{$\begin{array}{l}\text { Agotamiento } \\
\text { emocional }\end{array}$} & No & 52 & 93 \\
\hline & Sí & 4 & 7 \\
\hline \multirow{4}{*}{ Dimensiones } & Ninguna & 34 & 61 \\
\hline & Una & 19 & 34 \\
\hline & Dos & 3 & 5 \\
\hline & Tres & 0 & 0 \\
\hline \multirow{7}{*}{$\begin{array}{l}\text { Combinaciones } \\
\text { de dimensiones } \\
\text { positivas }\end{array}$} & Ninguna & 34 & 61 \\
\hline & Sólo agotamiento emocional & 2 & 3 \\
\hline & Sólo cinismo & 4 & 7 \\
\hline & Sólo ineficacia & 13 & 23 \\
\hline & Agotamiento emocional y cinismo & 1 & 2 \\
\hline & Agotamiento emocional e ineficacia & 1 & 2 \\
\hline & Cinismo e ineficacia & 1 & 2 \\
\hline
\end{tabular}

Valores de corte: agotamiento emocional $\geq 4,6$; cinismo $\geq 3,25$; ineficacia $\leq 4,6$.

yoría de los alumnos sin dimensiones, con sólo ineficacia y todos con agotamiento vivían más frecuentemente en Tlalpan o Coyoacán. Por igual, los alumnos con cinismo vivían en Tlalpan, Iztapalapa, Benito Juárez y Cuahtémoc. Los participantes con dos dimensiones del burnout vivían en delegaciones lejanas al sur de la ciudad (en donde se ubica la Facultad de Medicina); estas diferencias fueron significativas. El tiempo de traslado más frecuente fue de 31-60 min para los alumnos sin dimensiones (32\%). Por contra, todos los alumnos con sólo cinismo o sólo ineficacia reportaron > $61 \mathrm{~min}$; con agotamiento y cinismo, 61-120 min, y con ineficacia y otra dimensión, > $120 \mathrm{~min}$. Estas diferencias no fueron significativas. La mayor parte de los alumnos sin dimensiones (82\%) no habían sufrido violencia en el traslado, al igual que los que mostraron sólo ineficacia (85\%) y todos con cinismo y otra dimensión. En contraste, la mitad de los alumnos con sólo cinismo y todos con sólo agotamiento habían sufrido violencia en el traslado, mostrando significación estadística.

\section{Discusión}

Los alumnos del PECEM no presentaron burnout en el momento de la medición y la mayoría de ellos 
Tabla III. Distribución de las características de la población de estudio de acuerdo con las dimensiones del burnout $(n=56)$

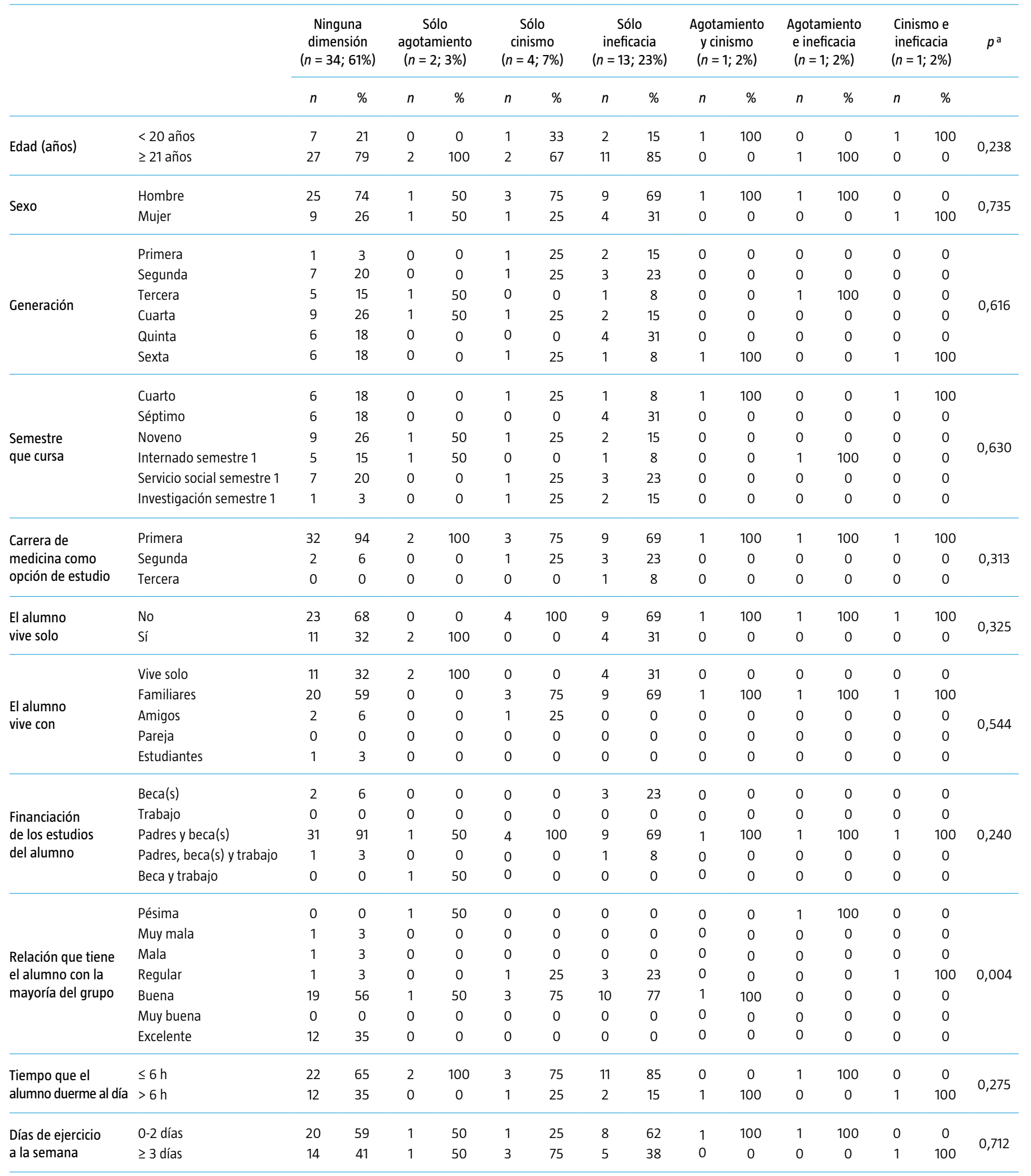


Tabla III. Distribución de las características de la población de estudio de acuerdo con las dimensiones del burnout $(n=56)$ (cont).

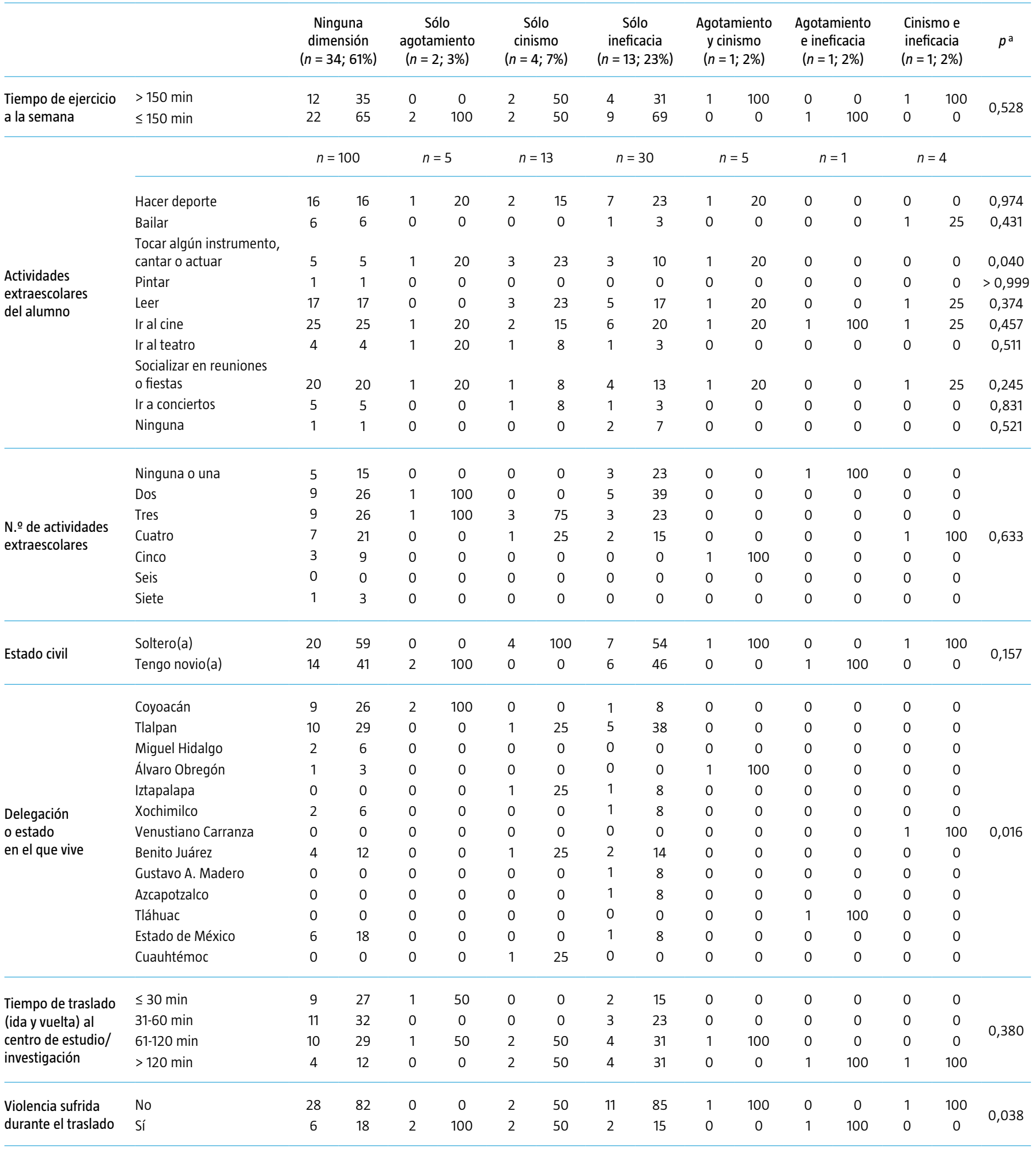

a Valor $p$ de la prueba exacta de Fisher. 
(61\%) tampoco presentó sus dimensiones. Este resultado es opuesto a lo que se ha comnunicado en estudiantes curriculares de medicina (45\% de burnout) [18]. El engagement es un concepto opuesto al burnout: la persona se siente capaz de responder a demandas laborales con eficacia, vigor (alta energía y resiliencia), dedicación (participación, entusiasmo, orgullo e inspiración) y absorción [2,19]. Al tornarse estas dimensiones en negativas, se llega al burnout $[2,20,21]$. Estudiantes universitarios con mejor desempeño, más preparados y con mejores expectativas de éxito son los que cuentan con engagement $[15,20,22,23]$. Asimismo, las estrategias para afrontar el burnout, como apoyo emocional externo (religión y social), evitación emocional y bajo nivel de espera al cambio, se relacionan con una baja frecuencia de dimensiones del síndrome (3,9\% agotamiento, $4,9 \%$ cinismo o ineficacia) $[3,13,24]$. De igual forma, los estudiantes que trabajan se perciben más autoeficaces y dedicados [24]. En contraste, el bajo vigor y el cinismo es común en alumnos con tendencia al abandono y hay relación con la baja satisfacción académica y las menores expectativas de éxito escolar [9,15,20,22-24]. En vista de que los estudiantes del PECEM tienen un alto desempeño (nota media $\geq 9$ ), se sugiere satisfacción, altas expectativas, administración efectiva del tiempo y uso de estrategias de afrontamiento, elementos que no fueron explorados en este estudio pero que posiblemente promovieron engagement en aquellos sin burnout o sus dimensiones.

Las dimensiones centrales del burnout son agotamiento y cinismo, ambas con una prevalencia en nuestro estudio del $7 \%$ y $11 \%$, respectivamente, más baja comparada con la comunicada en estudiantes de carreras de ciencias de la salud (hasta del 29\%) $[8,9,14,19,25]$. Los estudiantes con agotamiento sentían no poder dar más en sus tareas y podrían ser aquellos con peores expectativas de éxito y menor desarrollo profesional $[20,26]$. Esta dimensión se encontró en combinación con cinismo o ineficacia, situación cercana a presentar burnout. Para todos los alumnos con agotamiento, medicina fue su primera opción, la mayoría tenía $\geq 21$ años, empleaba más de una hora en el traslado, dormía poco y practicaba poco ejercicio, y comunicaba pocas actividades extraescolares. De interés fue encontrar que, contrariamente a alumnos con otras dimensiones, las variables más frecuentes en alumnos con agotamiento fueron: tener novio(a), violencia en los traslados $(p=0,038)$, vivían solos (Coyoacán, $p=0,006$ ), la relación con su grupo era pésima $(p=0,004)$ y el $50 \%$ pertenecían a la tercera, cuarta o sexta generación. Elementos del entorno académico se perciben como obstaculizadores o facilitadores del rendimiento académico: el obstáculo más frecuente era la relación extremadamente competitiva con los compañeros, y los facilitadores, el compañerismo, la familia y los amigos [23]. Es posible que, en los alumnos de nuestro estudio con agotamiento, las dificultades mencionadas y los traslados en la Ciudad de México contribuyeran al desarrollo de esta dimensión. El 11\% de los participantes mostraron cinismo, todos vivían acompañados, eran solteros(as) y el traslado suponía más de una hora (la mayoría sin violencia). La mayoría tenían $\geq 21$ años, medicina fue su primera opción y dormían poco. Contrariamente a aquellos con agotamiento, la mayoría tenían buena o regular relación con el grupo y mostraron una alta frecuencia de actividades extraescolares posiblemente en respuesta a la dimensión. La mitad pertenecían a la sexta generación (segundo año), etapa con alta carga académica y exámenes departamentales, que generan ansiedad, se visualizan como dificultades y se suman a las exigencias de permanencia en el PECEM [23]. Por otra parte, tocar un instrumento, cantar o actuar aparentemente son respuesta al agotamiento y al cinismo. Este dato apoya la utilidad de la materia optativa 'Arte y cultura' que se ofrece en la licenciatura de medicina, así como los conciertos informales que se organizan los viernes de 13 a 18 horas en la facultad [27]. Al contrario que en otros estudios, la dimensión más frecuente fue la ineficacia (27\%). Este resultado es similar al reportado en Colombia (prevalencia del 28\%) [12-14]. Esta dimensión es un sentimiento de incompetencia académica y en nuestros participantes es posible que existiera por intentar superar o mantener las expectativas de excelencia del programa [15]. La mayoría de los alumnos con ineficacia tenían $\geq 21$ años, solteros, de la quinta generación, vivían con familiares o solos, dormían poco, realizaban poco ejercicio y comunicaban más de una hora de traslado (sin violencia).

Entre los elementos causales del burnout se encuentran altas exigencias, dificultades materiales, condición física, estado de salud, estilo de vida, la personalidad y motivaciones para estudiar. Así, por el instrumento utilizado y las características de la población, la prevalencia del burnout académico varía ampliamente. Se ha encontrado burnout hasta en el 50\% de universitarios, con mayor frecuencia en estratos extremos de edad, solteros, sin hijos, con bajo rendimiento, con toma de medicación por los estudios y posibilidad de deserción [11-14]. En cuanto al sexo, la relación no es clara. Los resultados sugieren que las mujeres presentan menor frecuencia de dimensiones que los hombres $[11,13,22]$. La po- 
blación de alumnos del PECEM es principalmente masculina, una tendencia global de la menor participación femenina en ciencia y opuesto a la población mayoritariamente femenina en la Facultad de Medicina [28]. Así, no se observaron tendencias en las dimensiones del burnout de acuerdo con la variable sexo en el estudio. Otros aspectos relacionados con cinismo y agotamiento han sido el desempeño incompetente de los profesores y no ser la carrera la primera opción [13]. La carrera de medicina no fue primera opción para el $11 \%$ de los participantes en nuestro estudio, sin ser significativa la diferencia. Sin embargo, el $67 \%$ de los alumnos cuya primera opción no fue medicina mostraron cinismo $(25 \%)$ o ineficacia $(75 \%)$.

La importancia del estudio fue identificar burnout y sus dimensiones en estudiantes del PECEM. La descripción de las características de los alumnos que presentaron alguna dimensión y las variables que mostraron diferencias significativas con las dimensiones del burnout permitió identificar a aquellos en riesgo de padecer el síndrome. Los resultados obtenidos también serán útiles para diseñar estudios cualitativos en los cuales se investiguen las demandas contextuales que generan las dimensiones, se explore el impacto que tienen en la vida del estudiante y se identifiquen las estrategias que utilizan los alumnos para alcanzar sus metas académicas lidiando con el agotamiento, cinismo o ineficacia. Todo ello va encaminado a implementar medidas preventivas y estrategias de soporte, similares a las que se han creado en otros escenarios académicos [29-31].

Bibliografía

1. Universidad Nacional Autónoma de México, Consejo Universitario. Acuerdos (2011). URL: https://consejo.unam. $\mathrm{mx} /$ pleno/acuerdos. [19.03.2018].

2. Maslach C, Schaufeli WB, Leiter MP. Job burnout. Ann Rev Psychol 2001; 52: 397-422.

3. Rosales-Ricardo Y, Rosales-Paneque FR. Burnout estudiantil universitario: conceptualización y estudio. Salud Mental 2013; 36: 337-45.

4. Rosales-Ricardo Y, Rosales-Paneque FR. Hacia un estudio bidimensional del síndrome de burnout en estudiantes universitarios. Ciência \& Saúde Coletiva 2014; 19: 4767-75.

5. Pöhlmann K, Jonas I, Ruf S, Harzer W. Stress, burnout and health in the clinical period of dental education. Eur J Dent Educ 2005; 9: 78-84.

6. Salanova M, Cifre E, Grau R, Llorens S, Martínez IM. Antecedentes de la autoeficacia en profesores y estudiantes universitarios: un modelo causal. Revista de Psicología del Trabajo y de las Organizaciones 2005; 21: 159-76.

7. Carlotto MS, Nakamura AP, Câmara SG. Síndrome de burnout em estudantes universitários da área da saúde. Psico Porto Alegre 2006; 37: 57-62.

8. Barboza J, Beresin R. Burnout syndrome in nursing undergraduate students. Einstein (São Paulo) 2007; 5: 225-30.

9. Caballero CC, Abell R, Palacio J. Relación del burnout y el rendimiento académico con la satisfacción frente a los estudios en estudiantes universitarios. Avances en Psicología Latinoamericana 2007; 25: 98-111.
10. Macías AB. Escala unidimensional del burnout estudiantil. Investigación Educativa Duranguense 2008; 9: 104.

11. Bittar C. Burnout y estudios de personalidad en estudiantes universitarios [tesis] (2008). URL: http://fci.uib.es/digitalAssets/ 177/177915 2.pdf. [03.04.2018].

12. Castro-Bastidas CR, David-Ceballos OO, Ortiz-Delgado L. Síndrome de burnout en estudiantes de pregrado de la Universidad de Nariño. Revista Electrónica de Psicología Iztacala 2011; 14: 223-46.

13. Campos JADB, Jordani PC, Zucoloto ML, Bonafé FSS, Maroco J. Burnout syndrome among dental students. Revista Brasileira de Epidemiologia 2012; 15: 155-65.

14. Hederich-Martínez C, Caballero-Domínguez CC. Validation of Maslach Burnout Inventory-Student Survey (MBI-SS) in Colombian academic context. CES Psicología 2016; 9: 1-15.

15. Schaufeli WB, Martínez IM, Pinto AM, Salanova M, Bakker AB. Burnout and engagement in university students: a crossnational study. Journal of Cross-Cultural Psychology 2002; 33: 464-81.

16. Esteve EB, Salanova M, Schaufeli W, Nogareda C. NTP 732: síndrome de estar quemado por el trabajo 'burnout' (III). Instrumento de medición. Madrid: Instituto Nacional de Higiene y Seguridad en el Trabajo; 2007. URL: http:// www.insht.es/InshtWeb/Contenidos/Documentacion/ FichasTecnicas/NTP/Ficheros/701a750/ntp_732.pdf.

17. Maslach C, Jackson SE, Leiter MP. Maslach Burnout Inventory. Palo Alto, CA: Consulting Psychologists Press; 1986.

18. Dyrbye LN, Thomas MR, Huntington JL, Lawson KL, Novotny PJ, Sloan JA, et al. Personal life events and medical student burnout: a multicenter study. Acad Med 2006; 81: 374-84.

19. Schaufeli WB, Bakker AB. Job demands, job resources, and their relationship with burnout and engagement: a multi-sample study. Journal of Organizational Behavior 2004; 25: 293-315.

20. Martínez I, Salanova M. Niveles de burnout y engagement en estudiantes universitarios. Relación con el desempeño y desarrollo profesional. Revista de Educación 2003; 330: 361-84.

21. González-Romá V, Schaufeli WB, Bakker AB, Lloret S. Burnout and work engagement: independent factors or opposite poles? Journal of Vocational Behavior 2006; 68: 165-74.

22. Martínez-Martínez IM, Marques-Pinto A. Burnout en estudiantes universitarios de España y Portugal y su relación con variables académicas. Aletheia 2005; 21: 21-30.

23. Salanova-Soria M, Martínez-Martínez IM, Bresó-Esteve E Llorents-Gumbau S, Grau-Gumbau R. Bienestar psicológico en estudiantes universitarios: facilitadores y obstaculizadores del desempeño académico. Anales de Psicología 2005; 21: 170-80.

24. Palacio-Sañudo JE, Caballero-Domínguez CC, GonzálezGutiérrez O, Gravini-Gómez M, Contreras-Santos KP. Relación del burnout y las estrategias de afrontamiento con el promedio académico en estudiantes universitarios. Universitas Psychologica 2012; 11: 535-44.

25. Gorter RC, Eijkman MA, Hoogstraten J. Burnout and health among Dutch dentists. Eur J Oral Sci 2000; 108: 261-7.

26. Schaufeli WB, Salanova M, González-Romá V, Bakker AB The measurement of engagement and burnout: a two sample confirmatory factor analytic approach. Journal of Happiness Studies 2002; 3: 71-92.

27. Universidad Nacional Autónoma de México, Facultad de Medicina: oferta académica (2010). URL: http://oferta.unam. $\mathrm{mx} /$ carrera/archivos/planes/medicina-fmedicina-planestudio13. pdf. [03.05.2018].

28. Ramírez FO, Wotipka CM. Slowly but surely? The global expansion of women's participation in science and engineering fields of study, 1972-92. Sociology of Education 2001; 74: 231-51.

29. McCray LW, Cronholm PF, Bogner HR, Gallo JJ, Neill RA. Resident physician burnout: is there hope? Fam Med 2008; 40: 626-32.

30. Pedrals N, Rigotti A, Bitran M. Aplicando psicología positiva en educación médica. Rev Med Chil 2011; 139: 941-9.

31. Aherne D, Farrant K, Hickey L, Hickey E, McGrath L, McGrath D. Mindfulness based stress reduction for medical students: optimising student satisfaction and engagement. BMC Med Educ 2016; 16: 209. 